Pacific

Journal of

Mathematics

HEEGAARD SPLITTINGS OF HAKEN MANIFOLDS HAVE BOUNDED DISTANCE

Kevin HARTSHORN 


\title{
HEEGAARD SPLITTINGS OF HAKEN MANIFOLDS HAVE BOUNDED DISTANCE
}

\author{
Kevin HARTSHORN
}

\begin{abstract}
Given a Heegaard splitting and an incompressible surface $S$ and a Heegaard splitting of an irreducible manifold, I shall use a generalization of Haken's lemma proved by Kobayashi in order to define a pair of simple closed curves on the splitting surface such that each bounds a disc in one of the handlebodies of the splitting. By modifying the proof of Kobayashi's lemma, I shall show that the sequence of boundary compressions used to isotope $S$ places a bound on the distance between these two simple closed curves in the complex of curves. This will then place a bound on the distance of the Heegaard splitting.
\end{abstract}

\section{Introduction.}

Let $\Sigma$ be a closed, orientable surface of genus $g \geq 2$. Associated with $\Sigma$ is a "curve complex" $\mathcal{C}(\Sigma)$ that has been defined by Harvey [4]. A vertex of this complex is an isotopy class of essential simple closed curves on $\Sigma$. Two vertices are joined by an edge if the corresponding isotopy classes have disjoint representatives ${ }^{1}$.

Notation 1.1. Throughout this paper, I will use the notation $c$ or $c_{i}$ to denote a simple closed curve on the surface $\Sigma$, the isotopy class of that curve, or the corresponding vertex in the complex of curves. Generally, the distinction will be unimportant.

On this complex, we define the distance $d$ between two vertices - between two essential simple closed curves on $\Sigma$ - to be the minimum number of edges traversed to get from one vertex to the other. Essentially, the distance is simply the metric on the 1-skeleton of $\mathcal{C}(\Sigma)$ gotten by letting each edge have length 1. Recently, Hempel [6] and Masur and Minsky [9] independently showed that the diameter of $\mathcal{C}(\Sigma)$ is infinite.

Suppose that $\Sigma$ is the splitting surface for a Heegaard decomposition of a 3-manifold $M$. That is, suppose $M$ is decomposed by handlebodies $H_{1}, H_{2}$ such that $M=H_{1} \cup H_{2}$ and $H_{1} \cap H_{2}=\partial H_{1}=\partial H_{2}=\Sigma$. Let $\mathcal{C}_{i}(\Sigma) \subset \mathcal{C}(\Sigma)$

\footnotetext{
${ }^{1}$ In general, $\mathcal{C}(\Sigma)$ is a $(3 g-4)$-simplex such that each $k$ cell corresponds to a collection of $k+1$ disjoint (and distinct) isotopy classes.
} 
denote the subcomplex consisting of essential closed curves that bound a disc in $H_{i}$. Define the distance of the splitting to be

$$
d\left(H_{1}, H_{2}\right)=\min \left\{d\left(c_{1}, c_{2}\right) \mid c_{i} \in \mathcal{C}_{i}(\Sigma)\right\} .
$$

Hempel showed (again in [6]) for any $D$, there is a manifold $M$ that has a Heegaard splitting $\left(H_{1}, H_{2} ; \Sigma\right)$ with $d\left(H_{1}, H_{2}\right)>D$. Whether there is one manifold $M$ with splittings of arbitrarily large distance is not known (however, they would have to be non-Haken). Several results already known about Heegaard splittings can now be expressed in terms of this distance function:

- Reducibility implies distance 0: If $M$ contains an essential sphere, then for any Heegaard splitting of $M$, Haken's lemma [2, 7] shows that $d\left(H_{1}, H_{2}\right)=0$. Specifically, Haken's lemma states that the sphere can be positioned so that it intersects $\Sigma$ exactly once and bounds a disc in each handlebody. Notice that stabilization creates a splitting of distance 0 regardless of the original splitting's distance.

- Strong irreducibility and distance: Recall that a splitting $\left(H_{1}, H_{2} ; \Sigma\right)$ is called weakly reducible if there is a pair $c_{1}, c_{2} \subset \Sigma$ of essential simple closed curves with empty intersection such that $c_{i}$ is the boundary of a disc in $H_{i}$. A splitting that is not weakly reducible is called strongly irreducible. Thus weakly reducible is equivalent to $d\left(H_{1}, H_{2}\right) \leq 1$ and strongly irreducible is equivalent to $d\left(H_{1}, H_{2}\right) \geq 2$. A result of Casson and Gordon [1] shows that if $M$ contains a distance 1 splitting, then $M$ is Haken (a more specific description of the genus of the incompressible surface is given in $[5]$ ).

- Conversely, Hempel [6] proved that if $M$ contains an incompressible torus, then $d\left(H_{1}, H_{2}\right) \leq 2$ for any splitting $\left(H_{1}, H_{2} ; \Sigma\right)$.

In this paper, I will show the following generalization of the above results:

Theorem 1.2. Let $M$ be a Haken 3-manifold containing an orientable incompressible surface of genus $g$. Then any Heegaard splitting of $M$ has distance at most $2 g$.

In Section 2, I shall establish the main definitions and lemmas needed to prove the theorem. The theorem itself will be proven in Section 3.

\section{Euler characteristics and $\partial$-compressions.}

A surface $S$ embedded in a 3-manifold $M$ is called compressible if there is a simple closed curve $c \subset S$ such that $c$ bounds a disc in $M$, but not in $S$. Otherwise $S$ is called incompressible. A Haken manifold is an irreducible manifold containing an incompressible surface of genus $g \geq 1$. If $(S, \partial S) \subset(M, \partial M)$ is a surface in a manifold with boundary, then $S$ is called $\partial$-commpressible (boundary compressible) if there is an $\operatorname{arc} \beta \subset \partial M$ and an 
$\operatorname{arc} \alpha \subset S$ essential in $S$ such that $\beta \cap S=\beta \cap \alpha=\partial \beta=\partial \alpha$ with the property that $\alpha \cup \beta$ bounds a disc in $M \backslash S$. Otherwise, $S$ is called $\partial$-incompressible (boundary incompressible). Note that if $S$ is $\partial$-commpressible, then we can "compress" along the arc $\alpha$ to simplify the surface.

Let $M$ be a closed, orientable, irreducible 3-manifold. Let $\left(H_{1}, H_{2} ; \Sigma\right)$ be a Heegaard splitting for $M$. Suppose $S$ is a given closed, orientable, incompressible surface in $M$. Note that if $M$ contains an incompressible surface of positive genus, then the statement of Theorem 1.2 allows the distance of the splitting to be at least 2 . Thus I shall assume that the splitting $\left(H_{1}, H_{2} ; \Sigma\right)$ is strongly irreducible, and that the genus of $S$ is positive.

Kobayashi [8] proved the following generalization of Haken's lemma:

Lemma 2.1. If $M=\left(H_{1}, H_{2} ; \Sigma\right)$ is a strongly irreducible splitting of an irreducible manifold, then any closed, orientable, incompressible surface $S$ is ambient isotopic to a surface $S^{\prime}$ such that:

1) $S^{\prime}$ intersects $\Sigma$ transversely,

2) $S^{\prime} \cap H_{1}$ contains exactly one disc component,

3) $S^{\prime} \cap \mathrm{H}_{2}$ does not contain any disc components.

Kobayashi used what Jaco [7] called an isotopy of type $A$ in order to prove this lemma ${ }^{2}$. Because I will be using a similar isotopy to prove Theorem 1.2, I shall describe it again here.

Suppose that $S \cap H_{1}$ is $\partial$-commpressible in $H_{1}$. Let $\alpha \subset S \cap H_{1}$ be an essential compressing arc and $\beta \subset \partial H_{1}=\Sigma$ be the arc such that $\alpha \cup \beta$ bounds a disc $D$ in $H_{1}$. To perform a $\partial$-commpression of $S$ from $H_{1}$ or an isotopy of type $A$, we isotope $S$ to $S^{\prime}$ by "chopping" into $S \cap H_{1}$ and pushing $\alpha$ through the disc $D$ and across $\Sigma$ (see Figure 1). In a similar way we describe $\partial$-commpressions of $S$ from $\mathrm{H}_{2}$.

Lemma 2.2. Suppose that $S^{\prime}$ is the image of $S$ after a $\partial$-commpression of $S$ from $H_{1}$. Then $\chi\left(S^{\prime} \cap H_{1}\right)=\chi\left(S \cap H_{1}\right)+1$.

Proof. To see this, notice that the effect of the $\partial$-commpression on $S \cap H_{1}$ is removal of a 1-handle. This 1-handle is homotopic to a 1-cell with Euler characteristic -1 , and so removal of this handle raises the Euler characteristic by 1 .

Recall that if a surface $S$ is decomposed by $S=X \cup Y$, then the Euler characteristic of $S$ is given by

$$
\chi(S)=\chi(X)+\chi(Y)-\chi(X \cap Y) .
$$

In our current case, we decompose $S$ by $S \cap H_{1}$ and $S \cap H_{2}$ and notice that all the intersections are circles (with zero Euler characteristic). Thus we see

\footnotetext{
${ }^{2}$ I find this terminology somewhat unintuitive and so I will usually refer to this isotopy as a boundary compression, or $\partial$-commpression.
} 

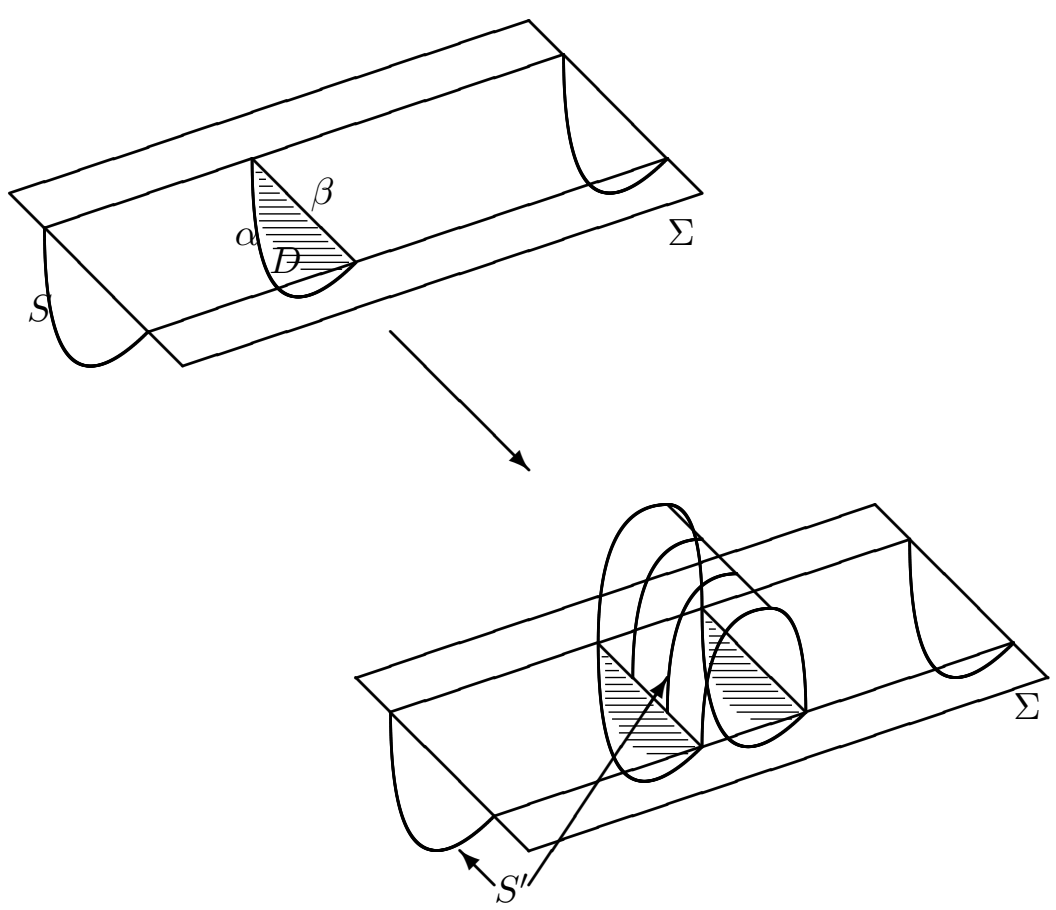

Figure 1

that

$$
\chi(S)=\chi\left(S \cap H_{1}\right)+\chi\left(S \cap H_{2}\right) .
$$

With this in mind, we deduce the following corollary to Lemma 2.2:

Corollary 2.3. Suppose that $S^{\prime}$ is the image of $S$ after an $\partial$-commpression of $S$ from $H_{1}$. Then $\chi\left(S^{\prime} \cap H_{2}\right)=\chi\left(S \cap H_{2}\right)-1$.

Remark 2.4. On the surface $\Sigma$, the effect of the $\partial$-commpression is surgery on a 1-submanifold:
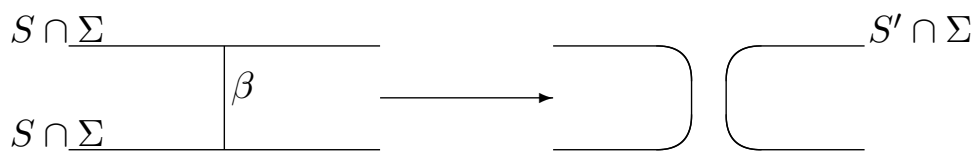

Notice that there are two possibilities for the arc $\beta$. Either it connects two essential simple closed curves on $\Sigma$ and forms a single simple closed curve after the surgery; or it connects two points of the same simple closed curve on $\Sigma$ to form two disjoint simple closed curves. The argument for this is given by Rubinstein and Scharlemann [11] to describe "saddle vertices". 
In order to prove Theorem 1.2, in the next section I will define an elementary compression and prove a few facts regarding the definition.

\section{Elementary compressions.}

When performing $\partial$-commpressions on $S \cap H_{1}$ (or similarly for $S \cap H_{2}$ ), there are generally choices for the compression arc $\alpha$ which are non-helpful. Specifically, suppose there is an annular component of $S \cap H_{1}$ parallel to $\partial H_{1}$ (such components will be referred to as $\partial$-parallel annuli). Then the $\partial$-commpression defined by a meridional arc $\alpha$ of this $\partial$-parallel annulus will leave a component of $S \cap H_{1}$ which is a disc parallel to $\Sigma$. In particular, the component of $S \cap \Sigma$ formed by this move will be inessential in $\Sigma$. As I am interested only in the components of $S \cap \Sigma$ which are essential in $\Sigma$, I would like to avoid this particular compression. However, the positioning of $S$ in $M$ may be such that the only possible $\partial$-commpressions are along $\partial$-parallel annuli.

To deal with this, I define a two step operation. The first is the removal of $\partial$-parallel annular components of $S \cap H_{1}$. Note that this compression along the meridional arc followed by pushing the resulting disc through $\Sigma$ will constitute the "removal" of a $\partial$-parallel annulus (by pushing it into $H_{2}$ ). This operation of "removing" a $\partial$-parallel annular component of $S \cap H_{1}$ will be referred to as an annular compression of $S$ from $H_{1}$.

The second step is a specific sort of $\partial$-commpression called an elementary compression. Define an essential compressing arc $\alpha$ of $S \cap H_{1}$ to be strongly essential if it is not the meridian of a $\partial$-parallel annular component of $S \cap H_{1}$. An elementary compression of $S$ from $W_{1}$ is a $\partial$-commpression of $S \cap H_{1}$ along a strongly essential arc $\alpha \subset S \cap H_{1}$.

Throughout this section, let $\left(H_{1}, H_{2} ; \Sigma\right)$ be a strongly irreducible Heegaard splitting of $M$ and $S$ an incompressible surface intersecting $\Sigma$ transversely. In the following lemmas, I shall list several helpful properties of both elementary and annular compressions. While all the lemmas in this section will refer to elementary and annular compressions from $H_{1}$, notice that they are equally true if the roles of $H_{1}$ and $H_{2}$ are reversed.

Lemma 3.1. Suppose $S \cap H_{1}$ is incompressible in $H_{1}$. If $\chi\left(S \cap H_{1}\right) \leq 0$, then there is an elementary or an annular compression of $S$ from $H_{1}$.

Proof. Because $S$ is incompressible in $M$, it must intersect $\Sigma$ nontrivially, and thus $S \cap H_{1} \neq \emptyset$. Further, the only incompressible, $\partial$-incompressible surfaces in a handlebody are discs. If all the components of $S \cap H_{1}$ were discs, then $\chi\left(S \cap H_{1}\right)$ would be positive. Thus, as $S \cap H_{1}$ is incompressible in $H_{1}$, there must be at least one component of $S \cap H_{1}$ which is $\partial$-commpressible, and so there is either an elementary compression of $S$ from $H_{1}$ or an annular compression of $S$ from $H_{1}$. 
Remark 3.2. Because any incompressible surface has a nonempty intersection with the Heegaard splitting surface, we can assume that $S \cap H_{1} \neq \emptyset$.

In Lemma 2.2, we saw how an elementary compression (a special case of $\partial$-commpression) affects the Euler characteristic of $S \cap H_{1}$. The following lemma provides the analogous statement for annular compressions.

Lemma 3.3. Suppose that $S^{\prime}$ is the image of an annular compression of $S$ from $H_{1}$. Then $\chi\left(S^{\prime} \cap H_{1}\right)=\chi\left(S \cap H_{1}\right)$, and $\chi\left(S^{\prime} \cap H_{2}\right)=\chi\left(S \cap H_{2}\right)$.

Proof. Note that the net effect of an annular compression is simply the removal of an annulus. Removing an annulus has no net effect on $\chi\left(S \cap H_{1}\right)$, as the Euler characteristic of an annulus is 0 . The comments leading to Corollary 2.3 show that there is also no change to $\chi\left(S \cap H_{2}\right)$.

Note that Lemmas 3.1 and 3.3 provide the following corollary:

Corollary 3.4. If $S \cap H_{1}$ is incompressible in $H_{1}$ and $\chi\left(S \cap H_{1}\right) \leq 0$, then (perhaps after some annular compressions) there is an elementary compression of $S$ from $H_{1}$.

Lemma 3.5. Suppose that $S \cap H_{1}$ is incompressible in $H_{1}$. Then the image $S^{\prime}$ of an elementary compression of $S$ from $H_{1}$ also has incompressible intersection with $H_{1}$.

Proof. Let the elementary compression of $S$ be defined by the disc $D \subset H_{1}$ with $\partial D=\alpha \cup \beta$, where $\alpha \subset S \cap H_{1}$ and $\beta \subset \Sigma-$ as described in Section 2 . Consider a small regular neighborhood of $D$ in $H_{1}$, say $D \times I$ (where $I=$ $[0,1])$. This can be chosen so $(\partial D) \times I=(\alpha \cup \beta) \times I$, where $\alpha \times I \subset S \cap H_{1}$ and $\beta \times I \subset \Sigma$. The effect of the elementary compression on $S \cap H_{1}$ is to replace the band $\alpha \times I$ by the pair of discs $D_{0}=D \times\{0\}$ and $D_{1}=D \times\{1\}$. So if $S^{\prime} \cap H_{1}$ is the image of $S \cap H_{1}$ after the elementary compression, we consider $D_{0}$ and $D_{1}$ as submanifolds of $S \cap W_{1}$.

Let $c \subset S^{\prime} \cap H_{1}$ be a simple closed curve such that $c=\partial \Delta$ for some disc $\Delta \subset H_{1} \backslash S^{\prime}$. We can isotope $c$ so that $c \cap D_{i}=\emptyset$ for $i=0,1$. Further, we can use an innermost disc argument (noting that $H_{1}$ is irreducible) to see that $\Delta \cap D_{i}=\emptyset$ for $i=0,1$. Thus by undoing the elementary compression, we can view $\Delta$ as a compressing disc for $S$. Since $S$ is incompressible in $H_{1}$, the curve $c \subset S \cap H_{1}$ must bound a disc in $S$. Because $c \cap D_{i}=\emptyset$, we see that the disc bounded by $c$ in $S$ must be disjoint from the strip $\alpha \times I$. Thus we can conclude that $c$ bounds a disc in $S^{\prime}$, and so $S^{\prime}$ is also incompressible.

Lemma 3.6. Suppose that $S \cap H_{1}$ is incompressible in $H_{1}$, that each component of $S \cap \Sigma$ is essential in $\Sigma$ and that $S^{\prime}$ is the image of an elementary compression of $S$ from $H_{1}$. Then each component of $S^{\prime} \cap \Sigma$ is essential in $\Sigma$. 
Proof. Assume there is a component $c^{\prime}$ of $S^{\prime} \cap \Sigma$ which bounds a disc in $\Sigma$. By the hypothesis of the lemma, $c^{\prime}$ must be a curve generated by the elementary compression of $S$. Consider the arc $\beta \subset \Sigma$ along which the boundary compression was defined. At this point we divide the proof into two cases, depending on whether $\beta$ joined together one or two components of $S \cap \Sigma$.

Case 1. Suppose that $\beta$ joined two points of the same component $c$ of $S \cap \Sigma$. Then $c$ is broken into two components $c^{\prime}, c^{\prime \prime}$ by the $\partial$-commpression and we assume $c^{\prime}$ bounds a disc $D$ in $\Sigma$. Suppose that $c^{\prime}=\partial D$ is as indicated below.

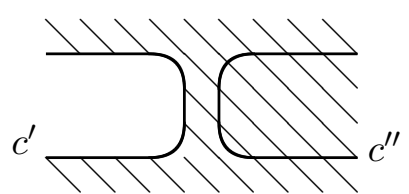

Then we see that $c^{\prime \prime} \subset D$. By the Jordan curve theorem, $c^{\prime \prime}$ must bound a disc in $D$, and thus in $\Sigma$. By looking at the preimage of this disc $D$ in $\Sigma$ before the elementary compression,

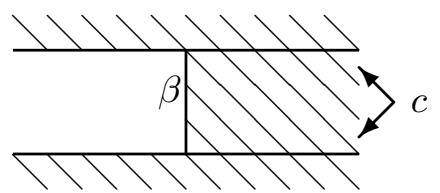

we see that the simple closed curve $c$ must bound a disc, contradicting the hypothesis of the lemma.

On the other hand, suppose that $c^{\prime}$ bounds the disc as indicated.

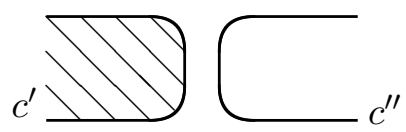

Then I claim that the $\partial$-commpression took place along an inessential arc $\alpha \subset S \cap H_{1}$. To see this, notice that on the preimage we have the picture:

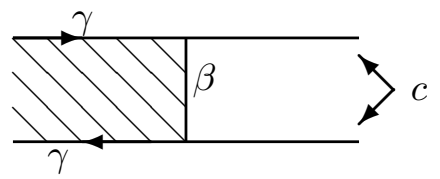

The curve $\beta \cup \gamma$ is isotopic to $c^{\prime}$ and thus bounds a disc in $\Sigma$. If $\alpha \subset S \cap H_{1}$ is the compressing arc for the elementary compression, then $\alpha \cup \beta$ bounds a disc in $H_{1}$. This implies that $\alpha \cup \gamma$ must also bound a disc in $H_{1}$. The simple closed curve $\alpha \cup \gamma$ can be homotoped to a simple closed curve in the interior of $S \cap H_{1}$. The incompressibility of $S$ in $H_{1}$ shows that this simple 
closed curve is not essential in $S \cap H_{1}$, and thus $\alpha$ is not an essential arc, contradicting the definition of an elementary compression.

Case 2. Suppose that $\beta$ joined two different components of $S \cap \Sigma$. In this case, both "components" of the image (near the arc $\beta$ )

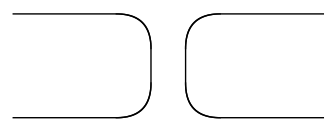

belong to the same curve $c^{\prime}$. There are then two ways in which $c^{\prime}$ can bound a disc:

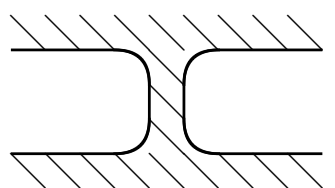

or
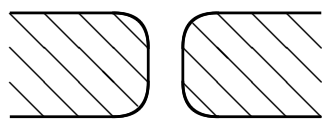

In the first case, the two curves comprising the preimage (before the elementary compression)

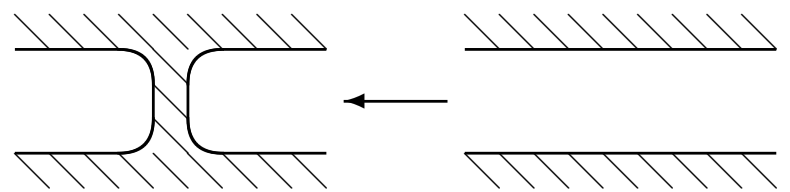

must also have bounded discs in $\Sigma$, contradicting the initial hypothesis.

In the second case, we see that the preimage

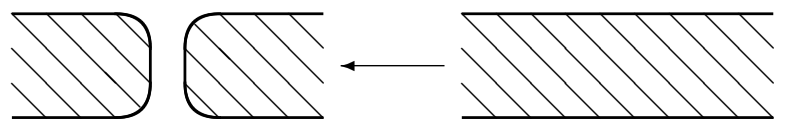

must have bounded an annulus in $\Sigma$. In addition, we can push $c^{\prime}$ into $H_{1}$ so it defines a compressing disc for $S^{\prime} \cap H_{1}$. By Lemma 3.5, we see that $c^{\prime}$ must be innessential in $S^{\prime} \cap H_{1}$. Thus we see that rather than an elementary compression, this must have been the first step of an annular compression.

Thus we see that $S^{\prime} \cap \Sigma$ consists only of simple closed curves which are essential in $\Sigma$.

Lemma 3.7. Suppose that $S \cap H_{1}$ is incompressible in $H_{1}$ and each component of $S \cap \Sigma$ is essential in $\Sigma$. If $S^{\prime}$ is the image of $S$ by an annular compression from $H_{1}$, then $S^{\prime} \cap H_{1}$ is incompressible in $H_{1}$ and each component of $S^{\prime} \cap \Sigma$ is essential in $\Sigma$. 
Proof. Because an annular compression simply deletes a component of $S \cap H_{1}$ (by moving it into $\mathrm{H}_{2}$ ), all the remaining components will still be incompressible in $H_{1}$. Similarly, the effect of the annular compression on the splitting surface $\Sigma$ is to delete two components of $S \cap \Sigma$, and thus all the remaining components of $S \cap \Sigma$ will still be essential in $\Sigma$.

Finally, we close the section with the lemmas that tie together the notion of distance and these elementary compressions:

Lemma 3.8. Suppose $S^{\prime}$ is the image of an elementary compression of $S$. Let $c \subset S \cap \Sigma$ and $c^{\prime} \subset S^{\prime} \cap \Sigma$ be any choice of components that are essential in $\Sigma$. Then $d\left(c, c^{\prime}\right) \leq 1$.

Proof. Suppose $c$ is not a component of $S \cap \Sigma$ affected by the elementary compression. Then $d\left(c, c^{\prime}\right) \leq 1$, as either $c \simeq c^{\prime}$ or $c \cap c^{\prime}=\emptyset$. Similarly, if $c^{\prime}$ is not in the image of the affected component(s) of the elementary compression, then $d\left(c, c^{\prime}\right) \leq 1$. So suppose $c$ is a component of $S \cap \Sigma$ affected by the elementary compression and $c^{\prime}$ is in the image of this $\partial$-commpression.

By considering the arc $\beta \subset \Sigma$ along which the elementary compression is defined,

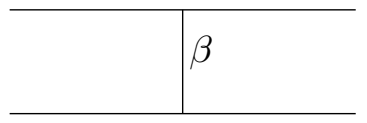

we can divide the proof into two cases, depending on whether $\beta$ connects one or two components of $S \cap \Sigma$.

Case 1. If $\beta$ connects two components $c_{a}, c_{b}$ of $S \cap \Sigma$, then we can choose normal directions on $c_{a}$ and $c_{b}$ in $\Sigma$ in such a way that near $\beta$ we have the picture below.

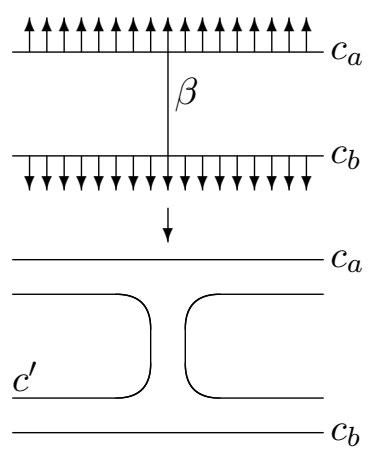

The image of this 1-surgery will be a single curve $c^{\prime}$ and this choice of a normal direction on $c_{a}, c_{b}$ in $\Sigma$ ensures that $c^{\prime} \cap c_{a}=c^{\prime} \cap c_{b}=\emptyset$. 
Case 2. Suppose that $\beta$ connects two points of the same component $c$ of $S \cap \Sigma$. Consider the compressing arc $\alpha \subset S \cap H_{1}$ for the boundary compression as well as the compressing disc $D$ with $\partial D=\alpha \cup \beta$. Because $S$ is orientable, we can choose a nonzero normal vector field $N(S)$ of $S \subset M$. If we then let $S_{\epsilon}$ be the image of $S$ after flowing in the direction $N(S)$ for a very short time, then $S_{\epsilon} \cap S=\emptyset$. Further, this normal direction can be chosen so that $S_{\epsilon} \cap D=\emptyset$. Note that on $\Sigma$, we then get the following picture.

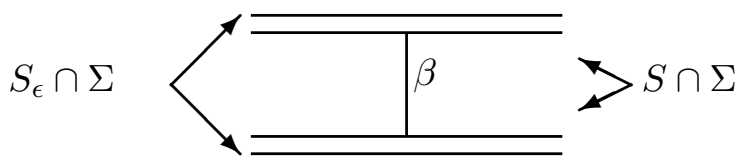

Let $c_{\epsilon} \subset S_{\epsilon} \cap \Sigma$ be the isotope of $c \subset S \cap \Sigma$. Then after the elementary compression of $S$, we see the intersections of $S^{\prime}$ and $S_{\epsilon}$ with $\Sigma$ as below.

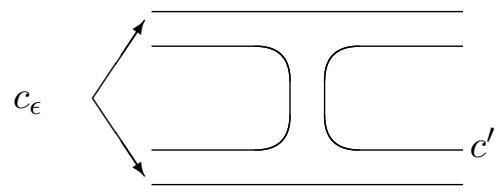

From this we see that even if $c \cap c \neq \emptyset$, the curve $c$ is isotopic to a curve $c_{\epsilon} \subset \Sigma$ which is disjoint from $c^{\prime}$. Thus $d\left(c, c^{\prime}\right) \leq 1$.

Lemma 3.9. Suppose that $S^{\prime}$ is the image of $S$ by an annular compression from $H_{1}$. Then the collection $S^{\prime} \cap \Sigma$ of simple closed curves is (up to isotopy) a proper subset of the collection $S \cap \Sigma$.

Proof. As in Lemma 3.7, this is due to the fact that annular compression simply removes two components of $S \cap \Sigma$, leaving the rest in place.

Together, Lemmas 3.8 and 3.9 will provide the means to place a bound on the distance $d\left(H_{1}, H_{2}\right)$. The idea in Section 4 will be to use a series of elementary compressions in order to develop a chain of essential simple closed curves on $\Sigma$, each distance 1 from the next. Because there may be $\partial$-parallel annuli in the way of this plan, some annular compressions may be needed at each stage. Lemma 3.9 ensures that this action will not affect the chain of essential curves.

\section{Proof of Theorem 1.2.}

Let $M$ be a closed, orientable, irreducible 3-manifold and suppose $S \subset M$ is a closed, orientable, incompressible surface of positive genus. Using an ambient isotopy, assume that $S$ meets $\Sigma$ transversely and minimally. That is, among all surfaces isotopic to $S$ in $M$, the number of components of $S \cap \Sigma$ 
is minimal. In most of the lemmas from Section 3, the embedded surface $S$ was required to have incompressible intersection with $H_{1}$ and/or have all components of $S \cap \Sigma$ essential in $\Sigma$. The following pair of lemmas show that these conditions are satisfied by placing $S$ in this minimal position with respect to $\Sigma$.

Lemma 4.1. Suppose that $S$ intersects $\Sigma$ minimally. Then for $i=1,2$, the intersection $S \cap H_{i}$ is incompressible in $H_{i}$.

Proof. Suppose that $S \cap H_{i}$ has a compressible component. Then there is a $\operatorname{disc}(D, \partial D) \subset\left(H_{i}, S \cap H_{i}\right)$ such that $\partial D$ does not bound a disc in $S \cap H_{i}$. Because $S$ is incompressible in $M$, there is a disc $D^{\prime} \subset S$ with $\partial D^{\prime}=\partial D$. Note that $D^{\prime} \cap \Sigma \neq \emptyset$. Because the manifold $M$ is irreducible, the sphere $D \cup D^{\prime}$ bounds a ball in $M$. Thus the surface $S^{\prime}=\left(S \backslash D^{\prime}\right) \cup D$ is isotopic to $S$ and $S^{\prime} \cap \Sigma$ has strictly fewer components than $S \cap \Sigma$.

Lemma 4.2. If the number of components of $S \cap \Sigma$ is minimal, then each component of $S \cap \Sigma$ is a simple closed curve which is essential in $\Sigma$.

Proof. Suppose $c \subset S \cap \Sigma$ is non-essential on $\Sigma$. Then let $D \subset \Sigma$ be the disc with $\partial D=C$. The incompressibility of $S$ in $M$ provides a disc $D^{\prime} \subset S$ with $\partial D^{\prime}=c$. Then the surface $S^{\prime}=\left(S \backslash D^{\prime}\right) \cup D$ is isotopic to $S$ as $M$ is irreducible. We can then push the closed disc $D \subset S^{\prime}$ slightly off $\Sigma$ so that $S^{\prime} \cap \Sigma$ has strictly fewer components than $S \cap \Sigma$.

Lemma 4.3. If the number of components of $S \cap \Sigma$ is minimal, then there are no d-parallel annular components of either $S \cap \mathrm{H}_{1}$ or $\mathrm{S} \cap \mathrm{H}_{2}$.

Proof. This follows as a direct consequence of Lemma 3.9.

The crux of the main theorem lies in the following pair of lemmas. The idea for the first lemma is to assume that $S \cap \Sigma$ is in minimal position and $S \cap H_{2}$ already has disc components of intersection. I want to move $S$ across $\Sigma$ until there is only one disc component. From that point, I will count the number of elementary compressions it takes to move $S$ further across $\Sigma$ to where $S \cap H_{1}$ contains a disc component.

Lemma 4.4. Let $M=\left(H_{1}, H_{2} ; \Sigma\right)$ describe a strongly irreducible splitting of an irreducible manifold $M$. Suppose that $S \subset M$ is a closed, incompressible surface such that each component of $S \cap \Sigma$ is essential in $\Sigma$ and each component of $S \cap H_{1}$ is incompressible in $H_{1}$. If $S \cap H_{2}$ contains essential discs, then there is a sequence of isotopies

$$
S \simeq S_{0} \simeq S_{1} \simeq \cdots \simeq S_{k} \simeq S_{k+1} \simeq \cdots \simeq S_{n}
$$

of $S$ having the following properties:

- Each component of $S_{i} \cap \Sigma$ is essential in $\Sigma$ (and each $S_{i} \cap H_{1}$ is incompressible in $H_{1}$ ). 
- For any choice of components $c_{i}$ of $S_{i} \cap \Sigma, d\left(c_{i}, c_{i+1}\right) \leq 1$ for $0 \leq i<n$.

- For $0 \leq i \leq k, S_{i} \cap H_{2}$ contains disc components.

- $k \leq n-2$, and for $k+1 \leq i \leq n-1$, neither $S_{i} \cap H_{1}$ nor $S_{i} \cap H_{2}$ contains any disc components.

- $S_{n} \cap H_{1}$ contains a single disc component.

Proof. If there are any $\partial$-parallel annular components of $S \cap H_{1}$, use annular compressions to remove them and form $S_{0}$. Form $\widehat{S}_{1}$ be performing an elementary compression on $S_{0}$ from $H_{1}$. If $\widehat{S}_{1} \cap H_{1}$ has any $\partial$-parallel annuli, perform annular compressions on $\widehat{S}_{1}$ from $H_{1}$ to form $S_{1}$. Otherwise, let $S_{1}=\widehat{S}_{1}$. Continue in this way recursively to form $\widehat{S}_{i}$ and $S_{i}$ for $1 \leq i \leq n$. The first point then follows inductively from Lemma 3.6 and Lemma 3.7.

Suppose that $c_{i}$ is a component of $S_{i} \cap \Sigma$. Then by Lemma 3.8 we know $d\left(c_{i}, \widehat{c}_{i+1}\right) \leq 1$ for any component $\widehat{c}_{i+1}$ of $\widehat{S}_{i+1} \cap \Sigma$. Further, by Lemma 3.9, we know that the collection of components of $S_{i+1} \cap \Sigma$ is a subset of the collection of components of $\widehat{S}_{i+1} \cap \Sigma$. Thus for any choice $c_{i+1}$ of component of $S_{i+1} \cap \Sigma$, we get the bound $d\left(c_{i}, c_{i+1}\right) \leq 1$.

Let $k$ be the greatest number such that $S_{k}$ contains a disc component of intersection with $\mathrm{H}_{2}$. By noting Lemmas 2.2 and 3.3, we see that the Euler characteristic changes by exactly 1 through each stage. Thus if $k$ is the greatest number such that $S_{k} \cap H_{2}$ has a disc component, then $S_{k} \cap H_{2}$ must have exactly one disc component. Otherwise, $\chi\left(S_{k+1} \cap H_{1}\right) \geq \chi\left(S_{k} \cap\right.$ $\left.H_{1}\right)+2$. Similarly, $n$ is chosen to be the least number such that $S_{n}$ has disc intersection with $H_{1}$ and we see that $S_{n}$ has exactly one disc component of intersection with $H_{1}$. Again, from Lemma 2.2, we know that such an $n$ exists, as after a finite number of compressions, the Euler characteristic will be positive, forcing there to be a disc component.

Note that because the splitting is strongly irreducible, we cannot have the case where $S \cap H_{1}$ and $S \cap H_{2}$ both have disc intersections. Recall from the remarks in the introduction that if $k=n$, then the splitting is reducible. If $k=n-1$, then by applying Lemma 3.8, we would find that the splitting is weakly reducible. Thus the inequality $k \leq n-2$ is due to the fact that $\left(H_{1}, H_{2} ; \Sigma\right)$ is strongly irreducible.

If $S \cap H_{1}$ has disc intersections, then we can apply the above lemma with the roles of $H_{1}$ and $H_{2}$ reversed. If, however, neither $S \cap H_{1}$ nor $S \cap H_{2}$ have disc intersections when $S \cap \Sigma$ is minimal, then we need to restate the lemma slightly. The idea here is to boundary compress $S$ in both directions, until we reach a disc intersection on either side.

Lemma 4.5. Let $M=\left(H_{1}, H_{2} ; \Sigma\right)$ describe a strongly irreducible splitting of an irreducible manifold $M$. Suppose that $S \subset M$ is a closed, incompressible surface such that each component of $S \cap \Sigma$ is essential in $\Sigma$ and each component of $S \cap H_{i}$ is essential in $H_{i}($ for $i=1,2)$. If neither $S \cap H_{2}$ nor 
$S \cap H_{1}$ contains essential disc components, then there is a sequence

$$
S_{-m} \simeq S_{-m+1} \simeq \cdots \simeq S=S_{0} \simeq \cdots \simeq S_{n}
$$

of isotopic copies of $S$ having the following properties:

- Each component of $S_{i} \cap \Sigma$ is essential in $\Sigma$.

- $S_{n} \cap H_{1}$ contains a disc component, as does $S_{-m} \cap H_{2}$.

- For $-m+1 \leq i \leq n-1$, neither $S_{i} \cap H_{1}$ nor $S_{i} \cap H_{2}$ contains any disc components.

- For any choice of components $c_{i}$ of $S_{i} \cap \Sigma, d\left(c_{i}, c_{i+1}\right) \leq 1$, where $-m \leq i<n$.

Proof. For $i>0$, define $\widehat{S}_{i}$ and $S_{i}$ exactly as in Lemma 4.4, again noting that there is a least $n$ such that $S_{n} \cap H_{1}$ has a disc component. To define $\widehat{S}_{i}$ and $S_{i}$ for $i<0$, we use the same method, but with the roles of $H_{1}$ and $H_{2}$ reversed. Yet again, note that there is a least $m$ such that $S_{-m} \cap H_{2}$ has a disc component.

Now the proof for each of the points of the lemma is completely analogous to the proof of Lemma 4.4.

Remark 4.6. If the number of components of $S \cap \Sigma$ is minimal, then the hypotheses of either Lemma 4.4 or Lemma 4.5 must be satisfied, depending on whether $S \cap H_{1}, S \cap H_{2}$, or neither contains disc components.

In either case, the idea is actually to start the incompressible surface in the position described in the conclusion to Kobayashi's lemma (Lemma 2.1). From there, we use a sequence of elementary compressions (together with annular compressions as necessary) to move the incompressible surface across $\Sigma$ until it again satisfies Lemma 2.1, but with the roles of $H_{1}$ and $H_{2}$ reversed. We then merely need to count the number of elementary compressions needed.

Main Theorem. If $M$ is a Haken 3-manifold containing an orientable incompressible surface of genus $g$, then any Heegaard splitting of $M$ has distance at most $2 g$.

Proof. Recall that we have an irreducible manifold $M$ decomposed by a strongly irreducible splitting $\left(H_{1}, H_{2} ; \Sigma\right)$. A closed, incompressible surface $S$ lies in $M$ such that the intersection $S \cap \Sigma$ is transverse and has a minimal number of components. If $g$ is the genus of $S$, we wish to show that there are a pair of essential simple closed curves $c_{1}$ and $c_{2}$ in $\Sigma$ such that $c_{i}$ bounds a disc in $H_{i}$ and $d\left(c_{1}, c_{2}\right) \leq 2 g$.

First, suppose that $S$ in this starting minimal position satisfies the hypothesis of Lemma 4.4. That is, the intersection of $S$ with $H_{2}$ already contains disc components. Then the conclusion of the lemma provides (up to a relabeling of the indices) a sequence $S_{0}, \cdots, S_{\ell}$ such that $S_{0}$ has a single 
disc component of intersection with $H_{2}, S_{\ell}$ has a single disc component of intersection with $H_{1}$, and all components of $S_{i} \cap \Sigma$ are essential in $\Sigma$ for $0 \leq i \leq \ell$. Notice by Lemma 2.2 that $\chi\left(S_{i} \cap H_{1}\right)=\chi\left(S_{i-1} \cap H_{1}\right)+1$ for each $0<i \leq \ell$.

Note that by relabeling indices, an identical sequence can be constructed if $S \cap H_{1}$ (instead of $S \cap H_{2}$ ) contained disc components.

On the other hand, suppose $S$ in this starting minimal position does not have disc intersections with either $H_{1}$ or $H_{2}$. Then by relabeling the indexing of the sequence formed in Lemma 4.5, we again have a sequence $S_{0}, \cdots, S_{\ell}$ such that $S_{0} \cap H_{2}$ and $S_{\ell} \cap H_{1}$ each contain a single disc component and each component of $S_{i} \cap \Sigma$ is essential in $\Sigma$ for all $i$. Using both Lemma 2.2 and Corollary 2.3, we again see that $\chi\left(S_{i} \cap H_{1}\right)=\chi\left(S_{i-1} \cap H_{1}\right)+1$ for each $0<i \leq \ell$.

In any case, let $c_{2}$ be the boundary of the disc component of $S_{0} \cap H_{2}$ and let $c_{1}$ be the boundary of the disc component of $S_{\ell} \cap H_{1}$. We know that if we choose any components $\gamma_{i}$ of $S_{i} \cap \Sigma$, then $d\left(\gamma_{i}, \gamma_{i+1}\right) \leq 1$. By letting $\gamma_{0}=c_{2}$ and $\gamma_{\ell}=c_{1}$, we get the bound $d\left(c_{1}, c_{2}\right) \leq \ell$. Now it is left to show that this number $\ell$ is at most $2 g$.

Recall the remarks leading to Corollary 2.3 that the decomposition of $S$ into $S \cap H_{1}$ and $S \cap H_{2}$ gives the equation

$$
\chi(S)=\chi\left(S \cap H_{1}\right)+\chi\left(S \cap H_{2}\right) .
$$

Because each $S_{i}$ is isotopic to $S$, it is also true that

$$
\chi(S)=\chi\left(S_{0} \cap H_{1}\right)+\chi\left(S_{0} \cap H_{2}\right) .
$$

Since $S_{0} \cap H_{2}$ contains exactly one disc component, note that $\chi\left(S_{0} \cap H_{2}\right) \leq 1$. Substituting the well-known formula for $\chi(S)$, we see

$$
\begin{aligned}
\chi(S) & \leq \chi\left(S_{0} \cap H_{1}\right)+1 \\
1-2 g & \leq \chi\left(S_{0} \cap H_{1}\right) .
\end{aligned}
$$

Using the inductive equation $\chi\left(S_{i} \cap H_{1}\right)=\chi\left(S_{i-1} \cap H_{1}\right)+1$, it is now clear that $S_{2 g} \cap H_{1}$ is forced to have at least one disc component of intersection. Because we chose $\ell$ to be the least integer with this property, we finally conclude that

$$
d\left(H_{1}, H_{2}\right) \leq d\left(c_{1}, c_{2}\right) \leq \ell \leq 2 g .
$$




\section{References}

[1] A.J. Casson and C.McA. Gordon, Reducing Heegaard splittings, Topology Appl., 27(3) (1987), 275-283, MR 98c:57020, Zbl 0632.57010.

[2] W. Haken, Some results on surfaces in 3-manifolds, Studies in Modern Topology, Math. Assoc. Amer. (distributed by Prentice-Hall, Englewood Cliffs, N.J.), (1968), 39-98, MR 36 \#7118, Zbl 0194.24902.

[3] K. Hartshorn, Heegaard Splittings: The Distance Complex and the Stablization Conjecture, University of California, Berkeley, 1999.

[4] W.J. Harvey, Boundary structure of the modular group, in Riemann surfaces and related topics: Proceedings of the 1978 Stony Brook Conference' (State Univ. New York, Stony Brook, N.Y., 1978), Princeton Univ. Press, Princeton, N.J., (1981), 245251, MR 83d:32022, Zbl 0461.30036.

[5] B. He and F. Lei, On reduction complexity of Heegaard splittings, Topology Appl., 69(2) (1996), 193-197, MR 96m:57027, Zbl 0854.57015.

[6] J. Hempel, 3-manifolds as viewed from the curve complex, Topology, 40(3) (2001), 631-657, CMP 1838999.

[7] W. Jaco, Lectures on Three-Manifold Topology, American Mathematical Society, Providence, R.I., 1980, MR 81k:57009, Zbl 0433.57001.

[8] T. Kobayashi, Heights of simple loops and pseudo-Anosov homeomorphisms, in 'Braids (Santa Cruz, CA, 1986),' Amer. Math. Soc., Providence, RI, (1988), 327338, MR 89m:57015, Zbl 0663.57010.

[9] H.A. Masur and Y.N. Minsky, Geometry of the complex of curves. I: Hyperbolicity, Invent. Math., 138(1) (1999), 103-149, MR 2000i:57027, Zbl 0941.32012.

[10] Geometry of the complex of curves. II: Hierarchical structure, Geom. Funct. Anal., 10(4) (2000), 902-974, MR 2001k:57020, Zbl 0972.32011.

[11] H. Rubinstein and M. Scharlemann, Comparing Heegaard splittings of non-Haken 3-manifolds, Topology, 35(4) (1996), 1005-1026, MR 97j:57021, Zbl 0858.57020.

Received August 3, 2000 and revised January 16, 2001.

Department of Mathematics

University of California, Davis

DAVIS, CA 95616

E-mail address: khartsho@math.ucdavis.edu 